Original Article

\title{
A CONTROLLED RELEASE MICROSPHERE FORMULATION OF AN ANTI-DIABETIC DRUG AND CHARACTERIZATION OF THE MICROSPHERE
}

\author{
BIPLAB KUMAR CHAKRA, SAUMEN KARAN, BISWAJIT DAS, SOUVIK DEBNATH, TAPAN KUMAR CHATTERJEE
}

Department of Pharmaceutical Technology, Jadavpur University, Kolkata 700032. W. B., India

Email: tkchatterjee_81@rediffmail.com

Received: 25 May 2018 Revised and Accepted: 23 Aug 2018

\section{ABSTRACT}

Objective: Here the objective of this study was to prepare and characterize sustained release metformin loaded microsphere formulation which was prepared by $\mathrm{W}_{1} / 0 / \mathrm{W}_{2}$ emulsion solvent evaporation technique.

Methods: Guar gum and sodium alginate were used as a matrix building material, whereas ethyl cellulose was applied as a coating polymer. Here various formulations were prepared by changing the drug and guar gum ratio, and the subsequent drug entrapment efficiency (DEE) and drug release were compared and evaluated.

Results: Scanning Electron Microscopy (SEM) studies revealed spherical particles with a smooth appearance. Fourier-transform infrared spectroscopy (FTIR) showed there was no interaction between the ingredients in the final formulation. X-ray Diffraction (XRD) studies showed the emergence of polymorphic forms in the final formulation. The drug entrapment in the final drug loaded microsphere formulations was varied from $30-66.78 \%$. The drug release studies showed the continuous release of the drug through twelve hours. The optimized formulation (f2) found to release $71.5 \%$ of drugs at the end of the 12 th hour following zero order release kinetics.

Conclusion: The increase in gum concentration in the $W_{1}$ phase, which enhances viscosity in the $W_{1}$ phase, resulting in an increase in the drug entrapment up to an optimum level and a decrease in the release rate. So, it can prolong the action. So by using this tool, we can say that metformin loaded microsphere formulation would be a suitable pharmaceutical formulation for the treatment of diabetic patients in modern drug therapy for its prolonged action.

Keywords: Diabetes, Metformin, $\mathrm{W}_{1} / 0 / \mathrm{W}_{2}$ emulsion, Guar gum, Microspheres, Sustained-release formulation

(C) 2018 The Authors. Published by Innovare Academic Sciences Pvt Ltd. This is an open-access article under the CC BY license (http://creativecommons.org/licenses/by/4.0/) DOI: http://dx.doi.org/10.22159/ijpps.2018v10i10.27541

\section{INTRODUCTION}

Diabetes mellitus is a complex phenomenon resulting in a big number of setbacks throughout the world. It is estimated as one of the five major causes of death globally. Almost 150 million people are affected by Diabetes internationally [1].

Metformin, a white crystalline, hygroscopic powder of a 1, 1dimethyl biguanide analog used as an antidiabetic drug from times immemorial. It has a scientific name $\mathrm{N}$-N-dimethyl imido di-carbon amide diamide hydrochloride (fig. 1) [2].

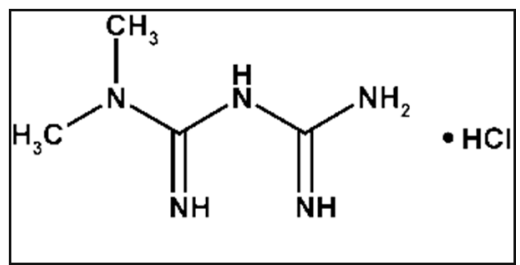

Fig. 1: Chemical structure of metformin hydrochloride

It reduces blood sugar by mainly blocking neoglucogenesis rather than increasing the secretion of insulin from the islets of Langerhans in the pancreas [3]. According to some experts, metformin is a drug of choice for newly diagnosed type- 2 diabetes and perhaps the best oral hypoglycemic agent proven to decrease cardiovascular mortality [4]. It is also used in polycystic ovary syndrome as it can reduce insulin resistance so as to produce ovulation and pregnancy [5]. The drug generally works by suppressing hepatic neoglucogenesis and decreasing glucose output in the liver. It also increases insulinmediated glucose disposal in muscle and fat. It increases the GLUT 1 transport from intracellular site to the plasma membrane. It also decreases the rate of intestinal absorption of glucose, other hexoses, amino acids, and Vit-B12. It increases peripheral glucose utilization by enhancing anaerobic glycolysis [6]. In diabetic patients, the blood sugar level should be maintained at a certain level for a long period. So, a sustained or controlled release dosage formulation will be beneficial for proper controlling of diabetes mellitus.

Microsphere formulations provide longer duration of drug action with minimal gastric irritation. As microsphere preparations contain the drug embedded in the matrix made of guar gum and sodium alginate, resulting in a continuous slow release through a longer period [7]. Metformin decreases blood glucose levels in the patients, those who do not produce insulin in appreciable quantity to control hyperglycemia [8]. So, the microspheres of metformin will be very helpful to fight against hyperglycemia for a longer duration [9].

Guar gum is used in food and pharmaceutical industries for longer times [10]. It is a natural nonionic polysaccharide obtained from the seeds of Cyamompsistetra tetragonolobus (Family-Leguminosae). It is hydrophilic in nature and produces its swelling property in cold water. Its gelling property is useful in producing a delay in the drug release so a better choice for controlled release formulations [11].

In this study, microsphere of metformin was prepared with an optimum concentration of all ingredients and guar gum was used as a matrix building material in combination with sodium alginate to provide a controlled and prolonged release for a longer time.

\section{MATERIALS AND METHODS}

\section{Materials}

Metformin was gifted from Stadmed Pharmaceuticals, Kolkata, India. Ethyl cellulose was purchased from Quest chemicals Kolkata, India. Dichloromethane, tween-80 and span 80 were purchased from 
Merck India. All other reagents were of analytical grade. Guar gum was collected from Bikaner district of Rajasthan, India.

\section{Animals}

A Sub-acute (14 d) toxicity study on Wistar rats was conducted according to the OECD 407 guideline (OECD, 2008). The maintenance of animal handling and care were performed under the guidelines of the Committee for the Purpose of Control and Supervision of Experiments on Animal (CPCSEA). Male Wistar rats (weight: 120-130 g) were selected for the toxicological evaluation of animals of both sexes were designated within the same weight range. Precautions were taken to ensure that the selected female animals were nulliparous and non-pregnant. The animals will be acclimatized under the standard controlled conditions, (temperature, $25 \pm 5 \mathrm{C}$; relative humidity, $55 \pm 10 \%$ ) with light and dark cycle of $14 \mathrm{~h}$ and $10 \mathrm{~h}$, respectively. A period of 7-10 d was provided to the animals to acclimatize under stipulated laboratory conditions prior to the initiation of experimentation. Throughout the experimental period, animals were housed in polypropylene cages provided by the standard pellet diet (Hindustan Lever Ltd., Mumbai, India) and water ad libitum [12].

\section{Methods}

\section{Preformulation studies}

\section{Preparation of stock solution}

The stock solution was prepared by dissolving $10 \mathrm{mg}$ of the drug in $50 \mathrm{ml}$ of distilled water kept in a $100 \mathrm{ml}$ of volumetric flask. Then the solution was made to $100 \mathrm{ml}$ by adding distilled water, and 100 $\mu \mathrm{g} / \mathrm{ml}$ stock solution was prepared.

\section{Determination of $\lambda$ max}

$10 \mu \mathrm{g} / \mathrm{ml}$ of drug solution was prepared from the stock solution. The absorbance in the UV spectrum was determined by scanning of a range $200 \mathrm{~nm}$ to $400 \mathrm{~nm}$, and maximum absorbance was found at $233 \mathrm{~nm}$ while distilled water was used as a blank. So the $\lambda$ max of the sample was $233 \mathrm{~nm}$.

\section{Preparation of standard curve}

Seven point calibration curve was prepared by spiking appropriate amounts of the stock solution into the corresponding buffer to obtain a final concentration of $5,10,15,20,25,30,35 \mu \mathrm{g} / \mathrm{ml}$ for the analysis. Absorbance was studied at $233 \mathrm{~nm}$, and a calibration curve was prepared by plotting absorbance against the concentration (fig. 2).

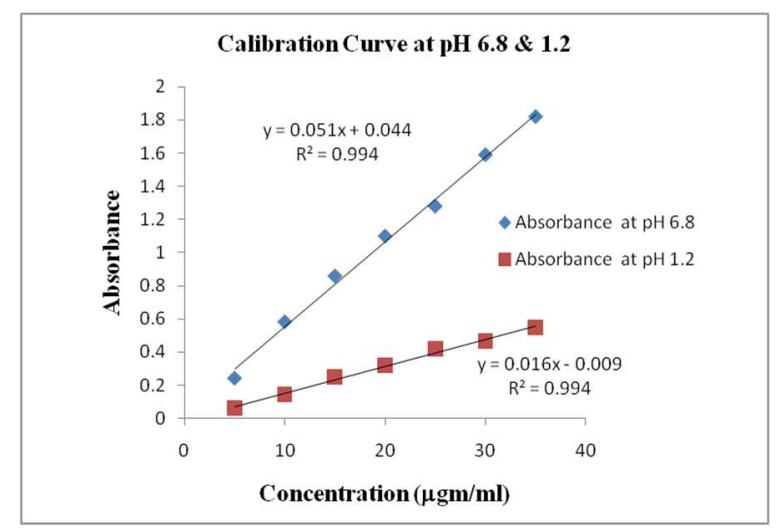

Fig. 2: Seven point calibration curve of metformin at $233 \mathrm{~nm}$ wavelength of $\mathrm{pH} 6.8$ and 1.2

\section{Swelling index}

$1 \mathrm{gm}$ of Guar gum was added in pH 6.8 buffer solution, $10 \mathrm{ml}$ of distilled water and $0.1 \mathrm{~N} \mathrm{HCl}$ solution. Then it was shaken for $10 \mathrm{~min}$ in a magnetic stirrer at high speed and allowed to stand for $24 \mathrm{~h}$. Swelling capacity was measured using the formula [13].

$$
\% \text { weight change }=\frac{\mathrm{Xv}-\mathrm{Xi}}{\mathrm{Xi}} \times 100
$$

\section{Microbial load study}

The pour plate method was applied to study the microbial and fungal load of the sample. Two sterilized Petri dishes were taken, and Soybean-Casein digests agar medium was poured into both the Petri dishes aseptically with fresh guar gum and ten months older sample respectively. After solidification of the media, both the Petri dishes were placed in the incubator at $37^{\circ} \mathrm{C}$ for one day. A total number of colonies were counted by the Coulter Counter method. In the same way, the total number of the fungal count was done by using potato dextrose agar medium [14].

\section{Toxicity study}

The subacute toxicity study was conducted on male Wistar rats (120-130 g). The solution of guar gum at a dose of 100, 200 and 400 $\mathrm{mg} / \mathrm{kg}$ body weight was administered orally to the three groups of six rats, respectively, for every day $14 \mathrm{~d}$ and control received a vehicle of the same volume. The toxic manifestation, i.e. body weight, mortality, and behavioral changes were regularly monitored. After $14 \mathrm{~d}$ all the surviving animals were fastened overnight and anesthetized with diethyl ether, and the heparinized blood samples were collected for determination of hematologic parameters and animals were sacrificed by euthanasia for the collection of internal organs kidney and liver. Then the collected organs were preserved in $10 \%$ formaldehyde solution for histopathological examinations. The research was conducted in accordance with the ethical rules on animal experimentation approved by the Ethical Committee, Department of Pharmaceutical Technology, Jadavpur University (Approval No: 147/1999/CPCSEA).

\section{Preparation of microsphere}

A small amount of sodium alginate was dissolved in $5 \mathrm{ml}$ of distilled water in a small beaker placed on a magnet stirrer. Guar gum was added to it with continuous stirring. Metformin as a weight of $80 \mathrm{mg}$ was added to it and stirring was done for almost half an hour by a magnetic stirrer. Then the preparation was taken with a 20 gauge needle. An organic solution was prepared with $1 \mathrm{gm}$ of ethyl cellulose and $30 \mathrm{ml}$ of dichloromethane (DCM). Then $300 \mu \mathrm{l}$ of span 80 was added to this organic solution. This preparation was transferred to the Homogenizer tube. The Homogenizer tube was subjected to rotation at approx 4500 RPM while the drug solution was poured drop by drop into it from the 20 gauge needle. A primary emulsion of W/O type was produced. A water phase was prepared with $100 \mathrm{ml}$ of distilled water and $200 \mu \mathrm{l}$ of tween 80 . The preparation was kept under a mechanical stirrer rotating at 780-800 RPM. The primary emulsion was then poured into the water phase drop wise by a 16 gauge needle and rotated for $3.5 \mathrm{~h}$ through mechanical paddle stirrer. Small spherical microspheres were formed which were filtered, air dried, weight and stored in a desiccator for future study [15].

\section{Characterization of prepared microspheres}

\section{Percentage Yield}

The percentage yield of Microsphere was calculated using the ratio of practical yield and theoretical yield. Practical yield is the weight of the microsphere obtained. Theoretical yield is the total weight of the raw materials [16].

Percentage of yield $=($ Practical yield $/$ Theoretical $) \times 100$

\section{Drug entrapment efficiency}

A small amount $(40 \mathrm{mg}$ ) of prepared microspheres were triturated properly and made into powder form, and then $100 \mathrm{ml}$ of phosphate buffer $(\mathrm{pH}$ 6.8) was added to it. This preparation was subjected to magnetic stirrer for $2 \mathrm{~h}$. Filtration of the solution was done by Whatman filter paper. $10 \mathrm{ml}$ of this stock solution was diluted with phosphate buffer (pH-6.8) and analyzed for metformin content at $233 \mathrm{~nm}$ [17].

$$
\begin{aligned}
& \text { Drug entrapment efficiency (DEE) } \\
& =\text { Experimental drug content } \\
& \text { /Theoretical drug content } \times 100
\end{aligned}
$$




\section{Particle size distribution and zeta potential}

The particle size distribution was obtained from the optical microscopic method. The mean average diameters of the microsphere particles were obtained in the SEM studies.

A weighed quantity of the experimental sample was dispersed in Milli-Q water (Milli-Q, Merck Millipore, Billerica, MA, USA) by vortexing and then sonicated and placed in a cuvette for zeta potential measurement [18].

\section{Scanning electron microscopy (SEM) analysis}

Particle size, shape and surface morphology were detected by SEM analysis. SEM was done by CARL ZEISS EVO 18 special edition machine with the platinum coating. The platinum coating was done by QUORUM Q150 TES machine [19].

\section{Fourier transform infrared spectroscopy (FTIR) study}

Fourier transform infrared spectroscopy (FTIR) was done to find out if there were any chemical interactions between the drug molecule and other ingredients used in the microsphere preparation. It was done on IR-Prestige-21, Shimadzu, Japan [20].

\section{X-ray diffraction (XRD) studies}

The samples of various batches were evaluated by X-ray diffraction studies. XRD studies were done by X-ray diffractometer of model no Ultima-111, Renuka (Japan), Cu target slide $10 \mathrm{~mm}$. The possible drug-polymer interaction was detected by XRD studies of the drug and the drug-loaded microsphere [21].

\section{Drug release study}

A drug release study was done in the dissolution test apparatus, LAB INDIA DS 8000 USP-type 2 (paddle type) apparatus calibrated at $37^{\circ} \mathrm{C}$ and rotated at $50 \mathrm{RPM}$. In the dissolution test apparatus initially, $750 \mathrm{ml}$ of acid buffer of pH-1.2 was added, and microspheres of $50 \mathrm{mg}$ were added into it. Then after $2 \mathrm{~h}, 150 \mathrm{ml}$ of trisodium orthophosphate buffer solution was added into the acidic solution so that the resultant $\mathrm{pH}$ of the solution became 6.8. From the final solution, $5 \mathrm{ml}$ of the sample was withdrawn in every 1 hour, and it was replaced by $5 \mathrm{ml}$ of phosphate buffer of $\mathrm{pH} 6.8$ every hour. The same thing was done at initial $2 \mathrm{~h}$ maintaining $\mathrm{pH}$ 1.2. This process was continued for $12 \mathrm{~h}$ [22].

\section{RESULTS}

\section{Swelling characteristics of the gum}

Swelling capacity of guar gum in distilled water, $0.1 \mathrm{NHCl}$ and $\mathrm{pH} 6.8$ buffers were described fig. 3 . Here the swelling index of the gum was slightly higher in $0.1 \mathrm{~N} \mathrm{HCl}$ than in buffer 6.8 and distilled water.

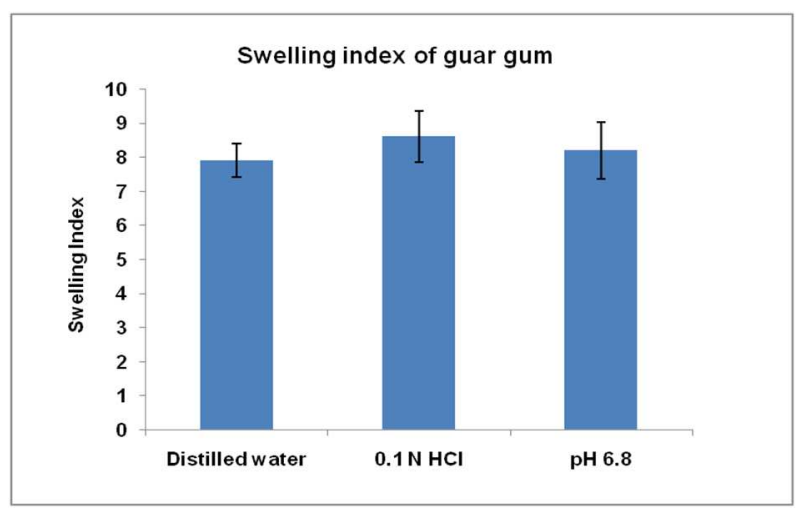

Fig. 3: Represents the swelling characteristic of guar gum at different $\mathrm{pH}$ (1.2 and 6.8). SD; Standard deviation of $n=3$

\section{Microbial load}

According to I. P. (4th edition, 1996), the microbial and fungal load should be less than $1000 \mathrm{CFU} / \mathrm{g}$ and $100 \mathrm{CFU} / \mathrm{g}$. The Microbial count was found to $290 \mathrm{CFU} / \mathrm{g}$ and $265 \mathrm{CFU} / \mathrm{g}$ for the older and the fresh sample of guar gum. The fungal count was found to be $75 \mathrm{CFU} / \mathrm{g}$ and73 CFU/g for the older and the newer sample of gum.

\section{Toxicity study of guar gum}

Treatment with guar gum at doses of 100,200 and $400 \mathrm{mg} / \mathrm{kg} \mathrm{b}$. w maintained the hemoglobin counts towards the normal levels. The RBC count was also maintained to a normal range on treatment with guar gum $(100,200$ and $400 \mathrm{mg} / \mathrm{kg} \mathrm{b.} \mathrm{w})$. Guar gum at an optimal dose of 200 and $400 \mathrm{mg} / \mathrm{kg} \mathrm{b.} \mathrm{w} \mathrm{slightly}$ brought down the WBC level. In the differential count, lymphocytes and monocytes were found to have minimal changes, and the neutrophils levels were little decreased in the drug-treated group when compared with the healthy group. Guar gum treatment at different doses as above significantly changed the relevant parameters approximately, to the normal values. Table 1 demonstrated the biochemical parameters SGOT, SGPT, SALP, and bilirubin not significantly changed $(100,200$ and 400 $\mathrm{mg} / \mathrm{kg}$ B.w.) as compared to a healthy control group. Results show platelets content also lightly increased dose-dependently in comparison to the normal.

Also in the histopathological studies (fig. 4) HandE stained sections of liver slices of healthy rat, shown in fig. 4, exhibiting the presence of all the normal features, including circular hepatic portal vein and a branch of the hepatic artery, as marked by arrows. The hepatocytes show prominent nuclei (fig. 4, marked by arrow) and the tissue section comprised hepatic sinusoids as usual. On the other hand, fig. 4 , none of the regular features as above mentioned could be found, rather it revealed extensive hepatocellular lesions, as pyknotic nuclei, exhibiting necrotic hepatocytes in guar gum treated groups. On treatment using test compound, the cellular features were found (fig. 4), some irregularities, e. g., deformity in the hepatic artery and irregular bile duct could be found. Along with this, guar gum treatment at a dosage of 100,200 and $400 \mathrm{mg} / \mathrm{kg} \mathrm{b}$. w, very least amount of phenotypic alteration and altered hepatocyte population were found (fig. 4), marked by the presence of healthy hepatocytes although a mild dilation of the central vein could be observed. On escalation of the dose to 200 and $400 \mathrm{mg} / \mathrm{kg} \mathrm{b}$. w, (fig. 4) the cellular and overall features were close to normal as had shown in marked by the presence of healthy hepatocytes, regular central vein, branch of the bile duct and hepatic artery exhibiting the superiority of the same. Histopathological evaluation of the normal kidney tissue of the rats demonstrated the normal structure of glomerulus surrounded by the Bowman's capsule, distal convoluted tubules and proximal convoluted tubules without any inflammatory alterations (fig. 4). The groups that were treated with guar gum at doses of 100,200 and $400 \mathrm{mg} / \mathrm{kg}$ b.w. demonstrated normal glomerulus, normal basement membrane, capillaries collecting ducts, tubules, ascending and descending loops without any inflammatory cells as compared to the normal healthy rat (fig. 4 marked by arrow).

\section{Percentage of yield and encapsulation efficiency}

The percentage yield and encapsulation efficiency of metformin were varied in all microsphere formulations. The percentage yield of microspheres varied from $42.20 \pm 0.83 \%$ to $94.84 \pm 1.88 \%$. It was also found that with the increase in the amount of guar gum in the internal phase the percentage yield was also increased. Due to the variation of drug and guar gum ratio, formulations were affected in table 2 . The loading was increased upto an optimum ratio of drug and guar gum after which an increase in the ratio this decreases the drug loading.

\section{Average particle size determination and zeta potential}

The particle size of the microspheres found to be increasing after a certain size range of $327.08 \mu \mathrm{m}$. After this optimum concentration (table 3), the particle size became larger with the increase in the amount of the guar gum. With the increase in the amount of guar gum in the solution resulted in an increase in the viscosity of the solution which provoked bigger particle formation. So after an optimum concentration of guar gum with the increase in the concentration of the guar gum particle size was also increased. 
Table 1: Effect of guar gum on blood hematological and biochemical parameter

\begin{tabular}{|c|c|c|c|c|}
\hline Parameters & Control & Guar gum (100 mg/kg) & Guar gum $(200 \mathrm{mg} / \mathrm{kg})$ & Guar gum $(400 \mathrm{mg} / \mathrm{kg})$ \\
\hline Hemoglobin (gm\%) & $14.41 \pm 0.023$ & $14.40 \pm 0.034^{* *}$ & $14.45 \pm 0.026^{* *}$ & $14.38 \pm 0.011^{* *}$ \\
\hline $\operatorname{RBC}\left(10^{6} / \mu \mathrm{l}\right)$ & $8.64 \pm 0.037$ & $8.62 \pm 0.089^{* *}$ & $8.71 \pm 0.201^{* *}$ & $8.85 \pm 0.056^{* *}$ \\
\hline $\mathrm{WBC}\left(10^{3} / \mu \mathrm{l}\right)$ & $13.73 \pm 0.068$ & $13.65 \pm 0.048^{* *}$ & $13.55 \pm 0.075^{* *}$ & $14.02 \pm 0.022 * *$ \\
\hline Neutrophil $\left(10^{3} / \mu \mathrm{l}\right)$ & $21.77 \pm 0.658$ & $22.01 \pm 0.358$ ** & $21.92 \pm 0.661^{* *}$ & $22.56 \pm 0.454^{* *}$ \\
\hline Monocyte $\left(10^{3} / \mu \mathrm{l}\right)$ & $2.33 \pm 0.347$ & $2.31 \pm 0.060 * *$ & $2.55 \pm 0.214^{* *}$ & $2.73 \pm 0.124^{* *}$ \\
\hline Lymphocyte $\left(10^{3} / \mu \mathrm{l}\right)$ & $73.56 \pm 0.347$ & $73.67 \pm 0.627^{* *}$ & $73.78 \pm 0.653^{* *}$ & $72.89 \pm 0.583^{* *}$ \\
\hline Eosinophil $\left(10^{3} / \mu \mathrm{l}\right)$ & $2.35 \pm 0.177$ & $2.32 \pm 0.748^{* *}$ & $2.57 \pm 0.432 * *$ & $2.21 \pm 0.654^{* *}$ \\
\hline Platelets $\left(10^{3} / \mu \mathrm{l}\right)$ & $1231.01 \pm 1.066$ & $1238.00 \pm 1.811^{* *}$ & $1241.00 \pm 0.713^{* *}$ & $1241.01 \pm 1.036^{* *}$ \\
\hline SGOT (U/l) & $88.99 \pm 0.323$ & $89.11 \pm 0.437^{* *}$ & $89.42 \pm 0.552 * *$ & $89.23 \pm 0.331^{* *}$ \\
\hline SGPT (U/l) & $32.63 \pm 0.541$ & $32.32 \pm 0.643^{* *}$ & $31.19 \pm 0.430$ ** & $33.14 \pm 0.473^{* *}$ \\
\hline
\end{tabular}

The each point represents the mean \pm SEM. ( $n=6$ mice per group). ${ }^{* *} \mathrm{p}<0.01$ statistically significant when compared with the normal saline group and drug-treated group.

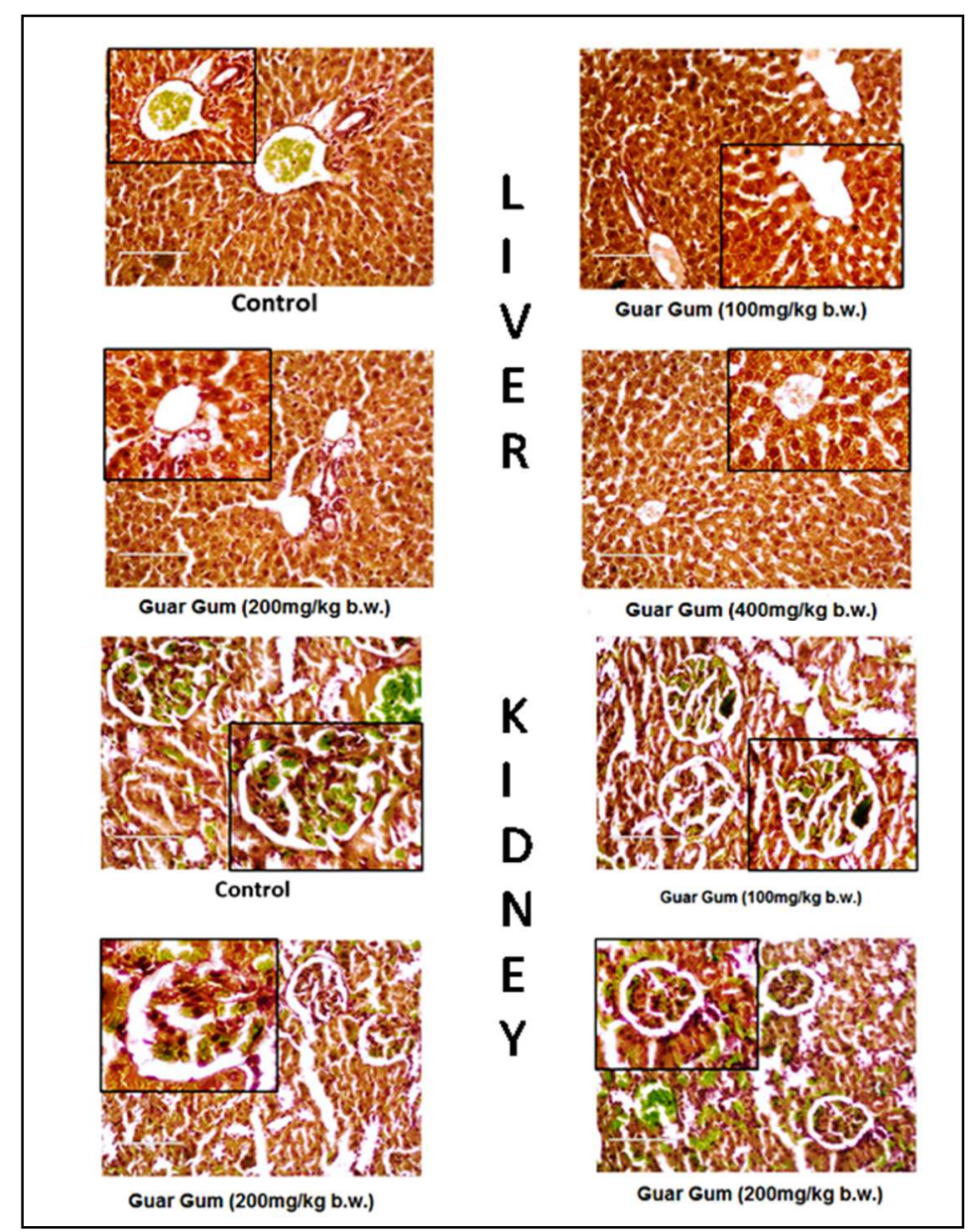

Fig. 4: Histopathological studies, HandE stained sections of liver and kidney slices of healthy rats. Liver slices are exhibiting the presence of all the normal features, including circular hepatic portal vein and branch of the hepatic artery, as marked by arrows. Kidney slices demonstrated normal glomerulus, normal basement membrane, capillaries collecting ducts, tubules, ascending and descending loops without any inflammatory cells as compared to the normal healthy rat (marked by the box). SD; Standard derivation for $n=6$

Table 2: Percentage of yield as well as drug entrapment efficiency of different formulations along with the different drug-gum ratio

\begin{tabular}{lll}
\hline Formulation code & Drug: guar gum & \% of yield \\
\hline F1 & $1: 0.22$ & $42.20 \pm 0.83$ \\
F2 & $1: 0.37$ & $49.16 \pm 1.27$ \\
F3 & $1: 0.50$ & $49.58 \pm 0.63$ \\
F4 & $1: 0.62$ & $49.65 \pm 0.56$ \\
F5 & $1: 0.75$ & $52.14 \pm 0.28$ \\
F6 & $1: 0.87$ & $89.38 \pm 1.32$ \\
F7 & $1: 1$ & $94.84 \pm 1.52$ \\
\hline
\end{tabular}

SD; Standard deviation for $n=3$ 
Table 3: The average particle size of drug-loaded microspheres with a different drug-gum ratio

\begin{tabular}{llll}
\hline Formulation code & Drug: guar gum & Average particle size $(\boldsymbol{\mu m})$ & Zeta potential(-mV) \\
\hline F1 & $1: 0.22$ & $327.08 \pm 2.45$ & $11.13 \pm 0.11$ \\
F2 & $1: 0.37$ & $392.08 \pm 1.96$ & $13.21 \pm 0.92$ \\
F3 & $1: 0.50$ & $397.78 \pm 3.61$ & $10.35 \pm 0.12$ \\
F4 & $1: 0.62$ & $540.42 \pm 2.55$ & $9.98 \pm 0.23$ \\
F5 & $1: 0.75$ & $548.77 \pm 1.39$ & $9.32 \pm 0.45$ \\
F6 & $1: 0.87$ & $553.48 \pm 5.38$ & $8.91 \pm 1.07$ \\
F7 & $1: 1$ & $556.48 \pm 4.15$ & $8.21 \pm 0.15$ \\
\hline
\end{tabular}

SD; Standard deviation for $n=3$

\section{Scanning electron microscopy (SEM) study}

The SEM was used to determine the particle size distribution, surface morphology, texture and also to examine the morphology of the fracture or sectioned surface of the prepared microspheres. The absence of pores in the microspheres was of major importance for the underlying drug release mechanisms because drug releases through the water-filled cavities were much faster than through dense polymeric matrix network. All microspheres were spherical in shape; smooth surfaced and had minuted pores on the surface. In (fig. 5) SEM studies indicated the spherical surface of the microsphere. The surfaces of the wall of the microspheres were rough and showing small holes due to the evaporation of the solvent. The surface of drug-loaded microspheres was found to be smooth with small pores whereas the surface of blank microspheres was rough having larger pores. In the study, the microsphere formulations with guar gum having smooth surface showed a slow and prolonged drug release profile as compared to the microspheres formulation without guar gum.

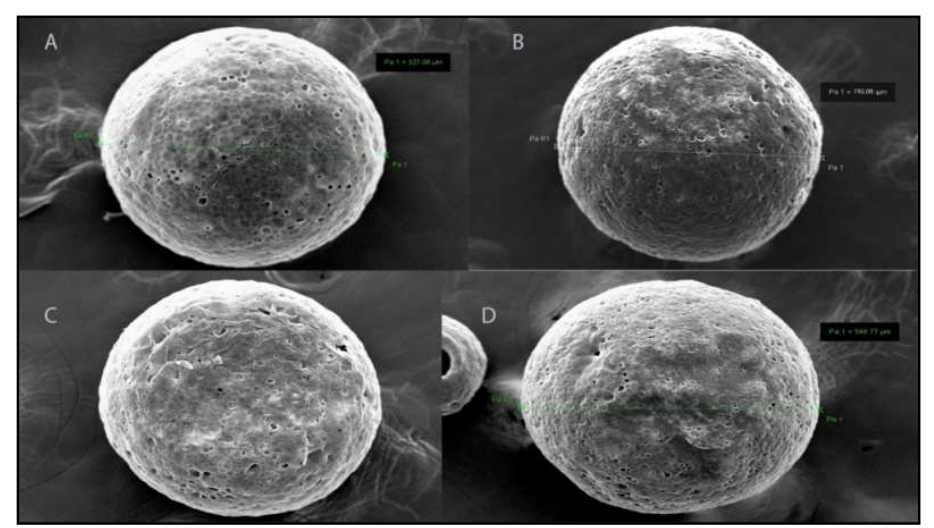

Fig. 5: A-Drug-loaded microspheres showing an apparently smooth surface, the existence of minute holes in the surface due to the rapid evaporation of the solvent in the magnetic stirrer. B-The best-optimized formulation (f2) showing smooth texture on the outer surface with a fine round shape in the appearance. Little roughness in the external surface helped its fine attachment with the intestinal lumen. $C$ -

The blank microsphere without the drug, having a spherical shape and rough in appearance. D-As, the concentration of the gum, increased in the internal phase so it produced a larger size due to the increase in viscosity in the W1 phase of W1/0/W2 microemulsion
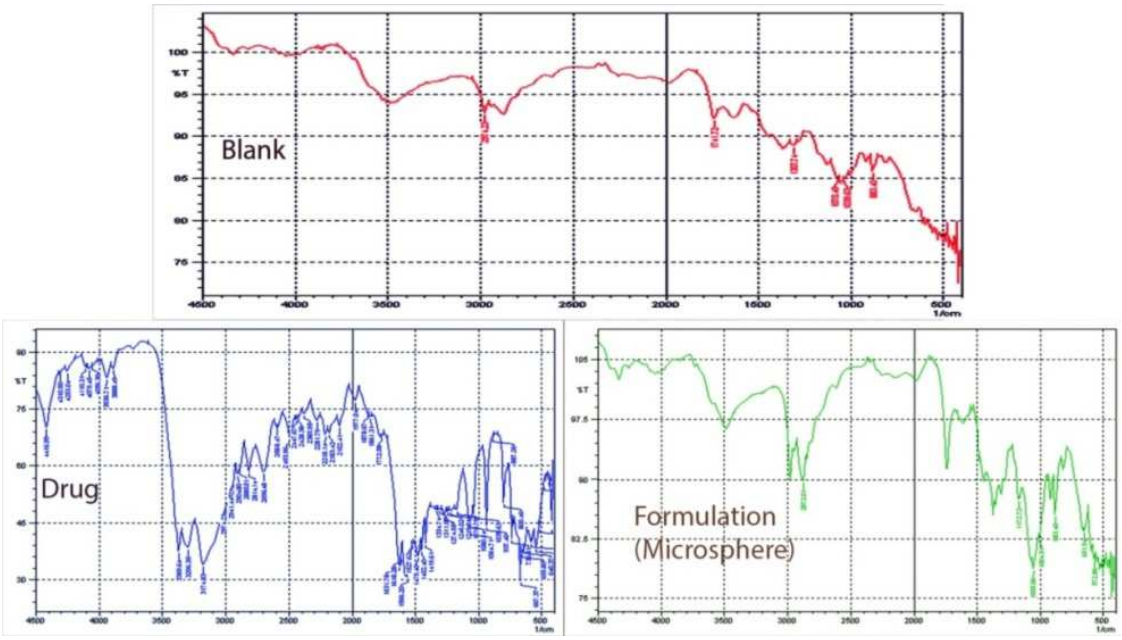

Fig. 6: 1. Blank-No peak was found at $3172 \mathrm{~cm}-1$ due to $\mathrm{N}-\mathrm{H}$ stretching, At $1065 \mathrm{~cm}-1$ no peak was found due to C-N stretching, At $1590 \mathrm{~cm}-$ 1 no peak was found due to $\mathrm{N}-\mathrm{H}$ bonding of the primary amine group. 2. Drug-At $3172 \mathrm{~cm}-1$ sharp peak due to $\mathrm{N}-\mathrm{H}$ stretching, At $1065 \mathrm{~cm}-$

1 peak was found due to $\mathrm{C}-\mathrm{N}$ stretching, At $1590 \mathrm{~cm}-1$ a peak was found due to $\mathrm{N}-\mathrm{H}$ bonding. 3. The formulation-almost similar peak at $3172 \mathrm{~cm}-1$ due to N-H stretching, (Microsphere) At $1065 \mathrm{~cm}-1$ a peak was found for C-N stretching, At $1590 \mathrm{~cm}-1$ a little bit similar peak was found due to $\mathrm{N}-\mathrm{H}$ bonding 


\section{Fourier transform infrared spectroscopy (FTIR) study}

FTIR was conducted to study the chemical interactions between the active ingredient metformin and all the other excipients used in the microsphere formulations. The entire observed spectrum is represented in fig. $\mathbf{6}$ where the characteristic peaks of drug-loaded microspheres were compared with that of the standard spectrum of metformin. From the FTIR studies, it was found that no chemical interaction occurred between the drug metformin and guar gum and polymer ethyl cellulose. The absorption peak of the drug found at $3172 \mathrm{~cm}-1$ due to $\mathrm{N}-\mathrm{H}$ stretching of the primary amine group (NH2). At $1065 \mathrm{~cm}-1$ due to C-N stretching a peak of metformin was found. At $1590 \mathrm{~cm}-1$ at peak due to $\mathrm{N}-\mathrm{H}$ bending of the primary amine group. The same type of peaks appeared (N-H stretching, C-N stretching and $\mathrm{N}-\mathrm{H}$ bending vibrations) in the spectra of guar gum containing microspheres of metformin. Although, similar characteristic peaks were not found in the blank microsphere formulations.

\section{XRD study}

According to the powder crystalline theory, the crystalline substances found to contain a well-defined structure. Atoms, ions, and molecules were arranged in a three-dimensional lattice which resulted in a tighter packing of components so well defined edges and faces diffract X-ray in a regular pattern. Amorphous solids had curved, and the irregular surface so did not provide well-resolved diffractogram. The powdered x-ray diffraction of metformin, drugloaded microsphere and guar gum were shown in the fig. 7. The diffraction pattern of pure metformin and metformin loaded microsphere showed a crystalline peak, but guar gum alone gave an amorphous peak. The result demonstrated that the crystalline peak of metformin and metformin loaded microsphere formulations were almost similar, but peak intensity diminishes in the formulation diffractogram due to dilution of the drug with gum and polymer. So, it can be said that the conducted XRD experiment also suggested that metformin was compatible with gum and other excipients.

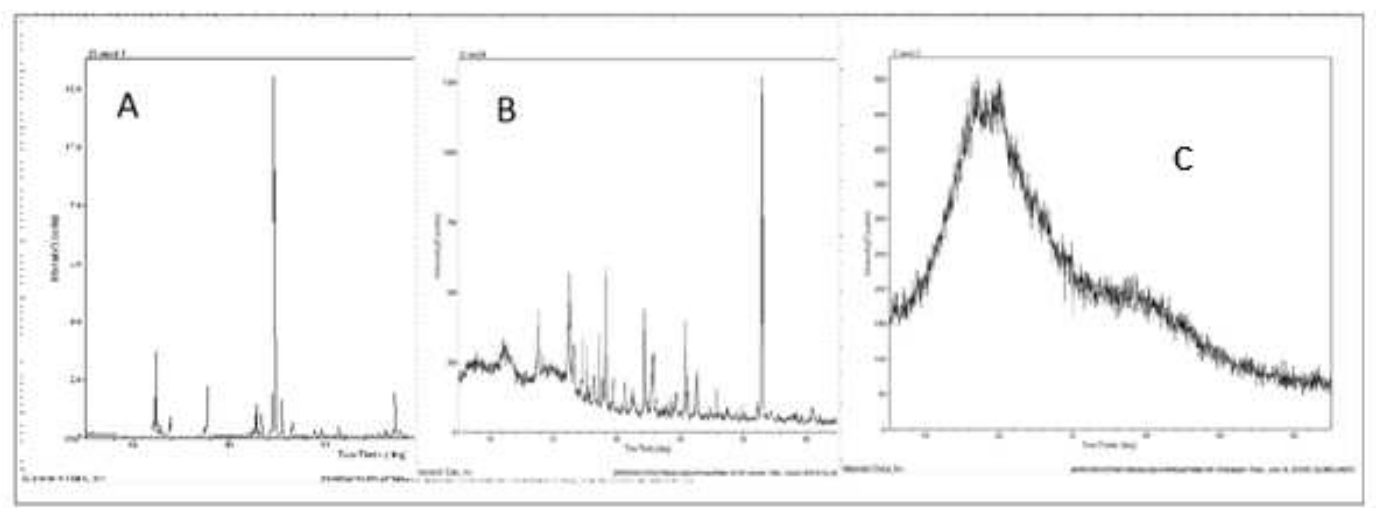

Fig. 7: A-Pure metformin introducing sharp crystalline peaks as there was no existence of polymorphic form. B-Metformin microsphere formulation containing both the drug and the excipients. Due to the presence of the drug crystalline peaks were found, whereas gums and excipients were responsible for the existence of polymorphic forms in the chromatogram. C-XRD study of gum showing the amorphous type of picture containing no crystalline peaks

\section{Dissolution study}

The mechanism of drug release from the microsphere formulations was biphasic in nature. Dissolution was carried out, and it was observed that the drug release from the microspheres in an acidic solution (pH-1.2) was slow in comparison to an alkaline solution (pH-6.8) (fig. 8). The collected drug release data from the dissolution test was evaluated using a number of kinetic models.

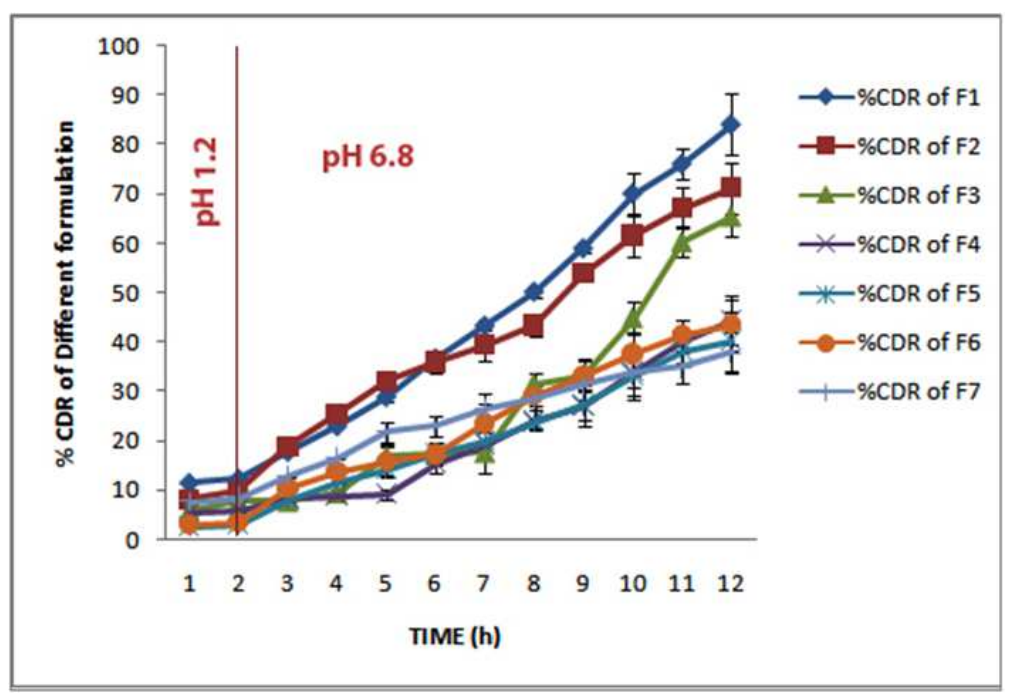

Fig. 8: Dissolution profile of $F 1$ to $F$ batches are depicted here in the figure. F1 batch is having the lowest concentration of gum showing the highest percentage of drug released (85\%). The optimized formulation F2 is showing $72 \%$ of drug released in $12 \mathrm{~h}$. With the concentration of the gum release of the drug from the microsphere was retarded. SD; Standard derivation for $\mathrm{n}=3$ 
The best-fitted model was analyzed by comparing the correlation coefficient values of different mathematical models in table 4 . From the recorded data the correlation coefficient value of drug loaded microsphere formulation ( $\mathrm{r} 2=0.99)$ was found to be higher and suitable according to the percentage of yield, the percentage of DEE and particle size than the other drug loaded microsphere formulations. The Zero Order kinetic model was the best satisfactory mathematical model for the microsphere formulation (f2).

Table 4: Drug release profile in dissolution media from different drug loaded microsphere formulations

\begin{tabular}{|c|c|c|c|c|c|c|c|c|c|c|c|}
\hline \multirow[t]{2}{*}{ Formulation code } & \multicolumn{2}{|c|}{ Zero-order } & \multicolumn{2}{|c|}{ First order } & \multicolumn{2}{|c|}{ Higuchi } & \multicolumn{3}{|c|}{ Korsmeyer-peppas } & \multicolumn{2}{|c|}{ Hixon crowell } \\
\hline & $\mathbf{K}_{\mathbf{0}}$ & $\mathbf{R}^{2}{ }_{0}$ & $\mathbf{K}_{1}$ & $\mathbf{R}^{2}{ }_{1}$ & $\mathbf{K}_{\mathbf{h}}$ & $\mathbf{R}^{2} h$ & $\mathbf{n}$ & Kkp & $\mathbf{R}^{2} \mathrm{kp}$ & $\mathbf{K}_{\mathrm{hc}}$ & $\mathbf{R}^{2} \mathrm{hc}$ \\
\hline F1 & 6.983 & 0.9833 & -0.064 & 0.8986 & 29.39 & 0.9175 & 0.8929 & 0.9008 & 0.9419 & 0.211 & 0.9949 \\
\hline $\mathrm{F} 2$ & 5.90 & 0.9905 & -0.045 & 0.957 & 26.87 & 0.9607 & 0.941 & 0.8245 & 0.9767 & 1.4521 & 0.9579 \\
\hline F3 & 5.423 & 0.8888 & -0.0369 & 0.8161 & 20.65 & 0.7687 & 0.9646 & 0.5794 & 0.8503 & 0.206 & 0.9557 \\
\hline F4 & 3.6936 & 0.9411 & -3.6936 & 0.9411 & 16.24 & 0.8537 & 0.926 & 0.5307 & 0.871 & 0.1745 & 0.9804 \\
\hline F5 & 3.547 & 0.9885 & -0.02 & 0.9009 & 16.00 & 0.9436 & 1.1988 & 0.3045 & 0.9757 & 0.1873 & 0.9618 \\
\hline F6 & 3.924 & 0.9879 & -3.924 & 0.9879 & 17.77 & 0.951 & 1.18 & 0.3742 & 0.9619 & 0.214 & 0.9765 \\
\hline F7 & 2.837 & 0.9831 & -0.0161 & 0.9917 & 13.117 & 0.9855 & 0.7119 & 0.8061 & 0.9702 & 0.1274 & 0.9384 \\
\hline
\end{tabular}

From the table, it is found that all the formulations released the drugs up to twelve hours. The best formulation F2 following zero-order drug release kinetics with almost $72 \%$ release of the drug

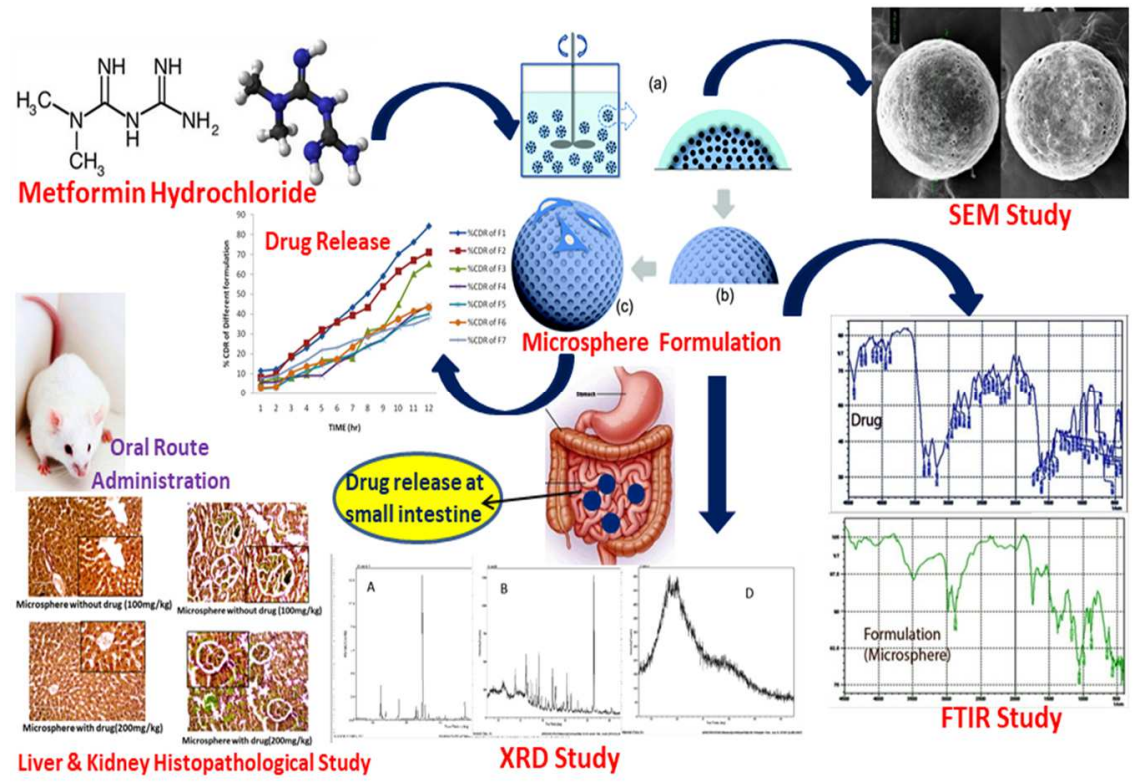

Fig. 9 Graphical abstract of metformin loaded microsphere

\section{DISCUSSION}

Guar gum and sodium alginate were used as matrix building materials whereas ethyl cellulose was applied as a coating polymer and these produced metformin loaded microsphere by $\mathrm{W}_{1} / \mathrm{O} / \mathrm{W}_{2}$ emulsion solvent evaporation technique. Initial experiments showed that a higher volume of the organic phase and internal aqueous phase as well as processing temperature considerably reduced DEE of the microsphere. If the volume of organic solvent increases, then the time required for evaporation of this solvent will be more which provides greater opportunity for the drug to partition from $W_{1}$ to $W_{2}$ phase. As a result, DEE of the microsphere decreased (66.78 to 35.75). A previous report represented that the entrapment efficiency of vitamin $B_{12}$ in poly ( $€$-caprolactone) microparticles decreased when the volume of external aqueous phase was increased and viceversa [23].

At the time of preparation of microsphere by $\mathrm{W}_{1} / \mathrm{O} / \mathrm{W}_{2}$ emulsionsolvent evaporation method, the organic phase separated the internal and external aqueous phases and acted as a diffusion barrier for the drug between the two aqueous phases. Higher the internal aqueous volume may increase the volume of $\mathrm{W}_{1}$ droplets in the oil phase and consequently may decrease the thickness of the organic polymer phase. This promoted more partitioning/leaching of the drug from internal to external aqueous phase. As a result, the DEE of the microcapsules decreased. The observation is in agreement with the results of other researchers [24-25]. In this research DEE also varied due to changes in the ratio of guar gum and these changes gave maximum DEE (66.78 \%) at a particular ration (drug: guar gum= 1:0.62) and decreased DEE followed by an increase in gum ratio. And also the percentage of yield of microsphere was proportionate with the gum ratio ( 94.4 at 1:1 drug: guar gum).

Increasing the volume of the internal aqueous phase $\left(\mathrm{W}_{1}\right)$ tended to increase the size of the microsphere [26]. Increase in volume of $W_{1}$ phase increased the number of dispersed droplets in a fixed volume of the organic phase, and the probability of coalescence between the dispersed droplets increased. This resulted in an increase in the size of the microsphere. Similar results had reported by various workers [27].

Incorporation of guar gum concentration in the $\mathrm{W} 1$ phase also affected the size of the matrix microsphere $(327.08 \mu \mathrm{m}$ to 556.48 $\mu \mathrm{m})$. An increase in the concentration of guar gum increased the size of the microsphere (table 5). As the concentration of guar gum was increased, the viscosity of the $\mathrm{W}_{1}$ phase also increased. This hindered easy breakdown of $\mathrm{W}_{1}$ phase into smaller droplets. In addition, an increase in viscosity of $W_{1}$ phase made the primary $\mathrm{W}_{1} / 0$ emulsion more viscous and formed larger $\mathrm{W}_{1} / 0 / \mathrm{W}_{2}$ emulsion droplets. As a result, the bigger size of microspheres were formed $(556.48 \mu \mathrm{m})$. 
Table 5: Parameter of the release of drug from the metformin loaded microsphere

\begin{tabular}{|c|c|c|}
\hline Formulation code & $\mathbf{t}_{50 \%}(\mathrm{~h})$ & $\mathbf{t}_{80 \%}(\mathrm{~h})$ \\
\hline $\mathrm{F}_{1}$ & 7.53 & 11.85 \\
\hline $\mathrm{F}_{2}$ & 8.38 & 12.23 \\
\hline $\mathrm{F}_{3}$ & 10.82 & ----b \\
\hline $\mathrm{F}_{4}$ & ----a & $----b$ \\
\hline $\mathrm{F}_{5}$ & $---a^{a}$ & $----b$ \\
\hline $\mathrm{F}_{6}$ & $---a^{a}$ & $----b$ \\
\hline $\mathrm{F}_{7}$ & $----a$ & $----b$ \\
\hline
\end{tabular}

---a Drug release was less than 50\% in 12 h.---b Drug release was less than $80 \%$ in $12 \mathrm{~h}$

The release of drug from the microsphere which was prepared with the different concentration of gum of the inner aqueous phase was slow. Replacement of the dissolution medium after $2 \mathrm{~h}$ with phosphate buffer (pH 6.8) produced a sudden increase in the release, which extended for different periods of time depending on the concentration of the gum in the W1 phase. Such a difference in release in the two dissolution media may be attributed to the $\mathrm{pH}$-dependent solubility of the drug which was poorly soluble in acidic solution and more soluble in an aqueous solution of higher $\mathrm{pH}$. In addition, as the concentration of the gum in the W1 phase was increased, the release of the drug in both the dissolution media decreased. The time required for 50\% ( $\mathrm{t} 50 \%)$ and $80 \%(\mathrm{t} 80 \%)$ drug release was determined from the cumulative percentage release versus time curves. Here t50\% was found to increase from $7.53 \mathrm{~h}$ to $10.82 \mathrm{~h}$, and $\mathrm{t} 80 \%$ increased from $16.35 \mathrm{~h}$ to $11.85 \mathrm{~h}$, as the concentration of gum in the internal aqueous phase increased that represented the microsphere had the capability to retard the release. That means it can be used as a sustained drug delivery formulation.

The higher the volume of $\mathrm{W}_{1}$ phase, increased the porosity of the wall of the microcapsules and resulted in faster drug release [28]. SEM photographs showed the presence of pores on the surface of the microcapsules. The development of pores may be due to leakage of water through the organic phase. During $\mathrm{W}_{1} / \mathrm{O} / \mathrm{W}_{2}$ emulsion solvent evaporation method, organic liquid diffuses from $\mathrm{W}_{1} / \mathrm{O}$ droplets to the external aqueous phase and simultaneously water from the external aqueous phase back diffuses into the droplets. The back diffusion was related to the difference in the osmolarity between the internal and external phases. The greater the back diffusion, the greater is the leakage of water [29] and hence, the wall of the microcapsules became more porous providing faster drug release.

The drug release properties of the microspheres showed a regular release pattern of the drugs up to twelve hours. The f2 batch having lowest content of guar gum showing a reasonable release of drug for sustained delivery. But as the guar gum concentration increases the release of the drug becomes lower, possibly due to the more viscous nature in the $W_{1}$ phase with the increase in the concentration of guar gum in the $W_{1}$ phase hindered the release of the drug [30].

\section{CONCLUSION}

In this research work, guar gum and sodium alginate were used as a matrix building material. Guar gum matrix microsphere of metformin, an anti-diabetic drug, was prepared by W1/0/W2 emulsion solvent evaporation method. Hematological, histopathological and toxicological data represented that guar gum is safe and nontoxic. With the increase in the guar gum concentration, it was found to entrap more amounts of drugs up to a certain extent, and the release of the drug is also dependent on the concentration of the guar gum in a sustained manner.

The release of the drug from the entire microsphere appeared to be biphasic releasing less amount of drug in $\mathrm{pH} 1.2$ and higher amount of drug in $\mathrm{pH}$ 6.8. The results of this study indicate that microcapsules prepared with guar gum as matrix material could be a suitable controlled release dosage form of metformin having high DEE that may release less amount of drug in stomach minimizing the emergence of gastric adverse effects and at the same time may provide a prolonged release in the intestine to achieve better drug therapy.

\section{ACKNOWLEDGMENT}

All India Council of Technical Education (AICTE)-Quality Improvement Program (QIP), New Delhi, India, sanctioned to Biplab Kumar Chakra through AICTE-QIP Research Fellowship scheme.

\section{AUTHORS CONTRIBUTIONS}

1st Author: Development of complete research plan, preparation and Drafting manuscript. 2nd Author: Helped to evaluate the analysis and interpretation of data and edit the complete manuscript. 3rd Author: Helped to edit and Critical revision of the article. 4th Author: Helped to edit the complete manuscript all tables and fig. preparation. $5^{\text {th }}$ Corresponding Author: Supervised, helped in data interpretation and manuscript evaluation.

\section{CONFLICT OF INTERESTS}

The author has no conflict of interest

\section{REFERENCES}

1. Tabish SA. Is diabetes becoming the biggest epidemic of the twenty-first century? Int J Med Health Sci 2007;1:V-VIII.

2. Beale JM, Block J, Hill R. Organic medicinal and pharmaceutical chemistry. Philadelphia: Lippincott Williams and Wilkins; 2010.

3. Diwedi Rohini, Alexandar A, Chandrasekar MJN. Preparation and in vitro evaluation of sustained release tablet formulations of metformin HCL. Asian J Pharm Clin Res 2012;5:45-8.

4. Harvey RA, Clark MA, Finkel R, Rey JA, Whalen K. Lippincott's illustrated reviews: pharmacology. Philadelphia: Wolters Kluwer; 2012.

5. Soyman Z, Daniel E Kel e klis. Polycystic ovary syndrome and metformin. J Obstetrics Gynaecol 2011;1:2.

6. Tripathi KD. Essentials of medical pharmacology. [ed.] M. Tripathi. 7th Edition. JP Medical Ltd; 2013. p. 275.

7. Ruhidas B, Naskar D, Banerjee S, Karan S, Chatterjee TK. Evaluation of gum katira as a model sustained release adjuvant in the preparation of etodolac loaded microsphere. Indian J Pharm Educ Res 2016;50:1.

8. Nethaji R, Narayanan A, Palanivelu M, Surendiran NS, Ganesan B. Formulation and evaluation of metformin hydrochloride loaded mucoadhesive microspheres. Int J Pharm Chem Biol Sci 2016;6:2.

9. Goswami S, Naik S. Natural gums and its pharmaceutical application. J Sci Innovative Res 2014;3:112-21.

10. Goldstein AM, Alter EN, Seaman JK. Guar gum. In Industrial Gums 1973;1:303-21.

11. Sharma S, Kaur J, Sharma G, Thakur KK, Chauhan GS, Chauhan K. Preparation and characterization of $\mathrm{pH}$-responsive guar gum microspheres. Int J Biol Macromol 2013;62:636-41.

12. Gorain B, Choudhury H, Tekade RK, Karan S, Jaisankar P, Pal TK. Comparative biodistribution and safety profiling of olmesartan medoxomil oil-in-water oral nanoemulsion. Regul Toxicol Pharmacol 2016;82:20-31.

13. Huanbutta K, Sittikijyothin W. Development and characterization of seed gums from tamarindus indica and cassia fistula as disintegrating agent for fast disintegrating Thai cordial tablet. Asian J Pharm Sci 2017;12:370-7.

14. Zaied SF, Youssef BM, Desouky O, El Dien MS. Decontamination of gum Arabic with $\gamma$-rays or electron beams and effects of these treatments on the material. Appl Radiat Isot 2007;65:26-31.

15. Pal T, Paul S, Sa B. Polymethylmethacrylate coated alginate matrix microcapsules for controlled release of diclofenac sodium. Pharmacol Pharm 2011;2:56-66.

16. Pavanveena C, Kavitha K, Kumar SA. Formulation and evaluation of trimetazidine hydrochloride loaded chitosan microspheres. Int J Appl Pharm 2010;2:11-4. 
17. kaliamurthi S, Selvaraj G. Insight on solid lipid nanoparticles: characterization and application in. J Critical Rev 2016;3:11-6.

18. Dang T, Cui Y, Chen YD, Meng XM, Tang BF, Wu J. Preparation and characterization of colon-specific microspheres of diclofenac for colorectal cancer. Trop J Pharm Res 2015;14:1541-7.

19. Venkateswaramurthy N, Sambathkumar R, Vijayabaskaran M Peruma. Clarithromycin mucoadhesive microspheres for antihelicobacter pylori therapy: formulation and in vitro evaluation. Int J Curr Pharm Res 2010;12:24-7.

20. Roy S, Manjanna KM. Effect of formulation variables on physicochemical characteristics and drug release properties of oral glipizide microspheres. J Adv Sci Res 2011;2:46-54.

21. Mankala SK, Nagamalli NK, Raprla R, Kommula R. Preparation and characterization of mucoadhesive microcapsules of gliclazide with natural gums. Stamford J Pharm Sci 2011;4:38-48.

22. Mandal AS, Biswas N, Karim KM, Guha A, Chatterjee S, Behera $\mathrm{M}$, et al. Drug delivery system based on the chronobiology-a review. J Controlled Release 2010;147:314-25.

23. Ramesh DV. Comparison of oil-in-oil, water-in-oil-in-water and melt encapsulation techniques for the preparation of controlled release B12 poly ( $€$-cprolactone) microparticles. Trends Biomaterials Artificial Organs 2009;23:21-3.

24. R Dinarvand SH, Moghadam A Sheikhi, F Atyabi. Effect of surfactant HLB and different formulation variables on the properties of poly-D,L-lactide micro-spheres of naltrexone prepared by double emulsion technique. J Microencapsulation 2005;22:139-51.

25. MK Yeh, SM Tung, DW Lu, CH Chiang. Formulation factors for preparing an ocular biodegradable delivery system of 5fluorouracil microparticles. J Microencapsulation 2001;18:50719.

26. Ganesh Subbiah, Kumar DS, Kumar BS, Abhilash R, Bharadwaj PS, Prudhviraj KVS, et al. Controlled release formulation and evaluation of idarubicin microsphere using biodegradable hydrophilic and hydrophobic polymer mixtures. Asian J Pharm Clin Res 2010;3:179-82.

27. MK Lai, RCC Tsiang. Microencapsulation of acetaminophen into poly(L-lactide) by three different emulsion solvent-evaporation methods. J Microencapsulation 2005;22:261-74.

28. Park G, Crotts TG. Protein delivery from poly (lactic-co-glycolic Acid) biodegradable microspheres: release kinetics and stability issues. J Micro-Encapsulation 1998;15:699-713.

29. DV Ramesh. Comparison of oil-in-oil, water-in-oil-in-water and melt encapsulation techniques for the preparation of controlled release B12 poly ( $€$-cprolactone) microparticles. Trends Biomater Artif Organs 2009;23:21-3.

30. Ong M, Li N, Sun S, Tiedt LR, Liebenberg W, de Villiers MM. Effect of viscosity and concentration of wall former, emulsifier and pore-inducer on the properties of amoxicillin microcapsules prepared by emulsion solvent evaporation. Il Farmaco 2005;60:261-7. 